\title{
Utility-Oriented On-Line Load Restoration Considering Wind Power Penetration
}

DOI:

10.1109/TSTE.2018.2846231

\section{Document Version}

Accepted author manuscript

Link to publication record in Manchester Research Explorer

\section{Citation for published version (APA):}

Zhao, J., Wang, H., Liu, Y., Azizipanah-abarghooee, R., \& Terzija, V. (2018). Utility-Oriented On-Line Load Restoration Considering Wind Power Penetration. IEEE Transactions on Sustainable Energy, 1-1.

https://doi.org/10.1109/TSTE.2018.2846231

\section{Published in:}

IEEE Transactions on Sustainable Energy

\section{Citing this paper}

Please note that where the full-text provided on Manchester Research Explorer is the Author Accepted Manuscript or Proof version this may differ from the final Published version. If citing, it is advised that you check and use the publisher's definitive version.

\section{General rights}

Copyright and moral rights for the publications made accessible in the Research Explorer are retained by the authors and/or other copyright owners and it is a condition of accessing publications that users recognise and abide by the legal requirements associated with these rights.

\section{Takedown policy}

If you believe that this document breaches copyright please refer to the University of Manchester's Takedown Procedures [http://man.ac.uk/04Y6Bo] or contact uml.scholarlycommunications@manchester.ac.uk providing relevant details, so we can investigate your claim.

\section{OPEN ACCESS}




\title{
Utility-Oriented On-Line Load Restoration Considering Wind Power Penetration
}

\author{
Jin Zhao, Hongtao Wang, Senior Member, IEEE, Yutian Liu, Senior Member, IEEE, \\ Rasoul Azizipanah-Abarghooee, Student Member, IEEE, and Vladimir Terzija, Fellow, IEEE
}

\begin{abstract}
An efficient load recovery of a bulk system with wind power penetration requires careful consideration of uncertainties related to it, as well as on-line data gathered from measurement devices. From the perspective of uncertainties, a novel utility-based decision-making method is proposed in this paper. The method combines both risk and return of load restoration strategy to assist decision-making in uncertain states, and it also provides utility function to present preference of load restoration strategy. Furthermore, a utility-oriented optimization model is created to select the strategy with the largest utility value with a certain confidence level. To achieve efficient utilization of on-line data, as well as to ensure high computation efficiency, the utilityoriented optimization is transformed into a scenario-based linear programming model. The proposed method fills the gap between on-line data and the optimal load restoration strategy in uncertain condition. Besides, the optimal strategy is provided with adjustable robustness according to security requirements and data exactness. Therefore, it is particularly applicable for on-line load restoration with wind power penetration. The effectiveness of the proposed method is validated using IEEE-30 bus test system and an actual power system from the North-East of Shandong province, China.
\end{abstract}

Index Terms - conditional value-at-risk, power system restoration, uncertain decision, wind uncertainty.

\section{NOMENCLATURE}

\section{A. Parameters}

$b_{i j} \quad$ Susceptance of grid line

c Risk-averse coefficient

$d \quad$ Distance between each tangent point

$\Delta f_{\max } \quad$ Maximum frequency deviation

$h \quad$ The number of hyperplane in cosine polyhedron

$g_{i j} \quad$ Conductance of gird line

$K \quad$ Parameter of cold load increment

$M \quad$ Scenario number

$m \quad$ Step number

$n \quad$ Total number of load cluster and wind power farm

$p_{i j, \max } \quad$ Maximum power flow of branch $i j$

$P_{0} \quad$ Normal load amount before blackout happen

$r_{i} \quad$ Ramp rate of generator $i$

$S_{j} \quad$ Capacity of generator $j$

$s, n-s \quad$ Number of load cluster and wind farm

This work was supported by the National Key R\&D Program of China (2016YFB0900100), (Corresponding author: Hongtao Wang)

J. Zhao, H. Wang and Y. Liu are with the Key Laboratory of Power System Intelligent Dispatch and Control of Ministry of Education (Shandong University), Jinan, China. (e-mail: hizhaojin@163.com; whtwhm@sdu.edu.cn; liuyt@ sdu.edu.cn)

R. Azizipanah-Abarghooee and V. Terzija are with the School of Electrical and Electronic Engineering, University of Manchester, Manchester, U.K. (e-mail: rasoul.azizipanah@manchester.ac.uk; vladimir.terzija @ieee.org).
$T_{\min }, T_{\max }$ Minimum, maximum time interval of one step

$t_{0}, \Delta t_{1}, \Delta t_{2}$ Time parameters for cold load pickup

$V_{\text {min }}, V_{\text {max }}$ Lower and upper limit of voltage

$\beta \quad$ Confidence level

$\theta_{\Delta}^{0} \quad$ Bound of the phase angle difference

$w_{i} \quad$ The weight of load $i$

$\varepsilon_{i} \quad$ Frequency response rate of generator $i$

B. Sets

$\boldsymbol{E}_{\boldsymbol{L}} \quad$ Set of load amount expectations

$\boldsymbol{E}_{\boldsymbol{W}} \quad$ Set of wind power output expectations

$\boldsymbol{G}, \boldsymbol{W}, \boldsymbol{L}$ Set of unit buses, wind farms buses and load buses

$\boldsymbol{N} \quad$ Set of nodes of the whole system

$\boldsymbol{P} \quad$ Input data matrix

$\boldsymbol{X}_{\boldsymbol{L}} \quad$ Set of load pick-up decision variables

$\boldsymbol{X}_{W} \quad$ Set of wind farm integration decision variables

$\boldsymbol{Y}_{\boldsymbol{L}} \quad$ Set of uncertain load amount variables

$\boldsymbol{Y}_{\boldsymbol{W}} \quad$ Set of uncertain wind power output variables

$\boldsymbol{w}_{L} \quad$ Set of load weights

C. Variables

$\cos ^{*} \theta_{i j} \quad$ A approximation value of $\cos \theta_{i j}$

$f, \Delta f \quad$ Frequency, frequency deviation

$P_{C L} \quad$ Cold load pick-up amount

$P_{G, i} \quad$ Output of unit $i$

$P_{W, s i g} \quad$ Sign of wind power available

$p_{i}, q_{i} \quad$ Active and reactive power at bus $i$

$p_{i j}, q_{i j} \quad$ Active and reactive power flow of branch $i j$

$t_{m} \quad$ Operation time of load pick-up in step $m$

$u_{k} \quad$ Auxiliary variable

$V_{i}, \Delta V_{i} \quad$ Voltage deviation of bus $i$

$\alpha_{\beta} \quad$ Threshold of uncertain power deficiency with $\beta$

$\delta \quad$ Difference between the target voltage and true value

$\theta_{i j} \quad$ Voltage angle difference of bus $i$ and bus $j$

$\Phi_{\beta} \quad$ Conditional expectation of power deficiency

\section{Functions}

$f($ ) Function of system uncertain power deficiency

$f_{\text {ris }}($ ) Risk function

$f_{\text {ret }}() \quad$ Return function

$P_{C L}() \quad$ Cold load pickup function

U( ) Function of the utility

$\rho$ ( ) Probability density function

$\Psi($ ) Cumulative distribution function

$\Phi_{\beta}(), F_{\beta}($ ) Functions of CPD

\section{INTRODUCTION}

$\mathrm{T}$ O ensure sustainable production of clean energy, the level of penetration of renewable energy sources, e.g. wind 
power, has been significantly increased over last few decades in the whole world [1]. However, the large-scale integration of intermittent wind power has an evident impact to power system restoration. In the event of European $4^{\text {th }}$ November 2006 disturbance [2], the uncontrolled wind power integration was resulted in the overloading of internal lines and exceeding of power transfer capacity margins. This problem led to an increased risk of a blackout. Besides, the unexpected shutdown of wind turbines, due to the extreme weather condition, is one of the causes for South Australia blackout in 2016 [3]. Obviously, the uncertainty of wind power has negative effects on power system restoration. Nevertheless, an unacceptable power imbalance could happen if the wind power is rejected in restoration process since it is indispensable in the future power systems. Additionally, through a reasonable planning, wind power can be regarded as a valuable resource with less start-up time constraint. It means that wind power can significantly contribute to the existing power system restoration procedure. In reality, restoration plans proposed in [4] and [5] provide some basic rules for the wind power application during restoration process, whereas the related rule is very rough. To satisfy both the security and efficiency of power system restoration procedures, more extensive studies should be undertaken.

Power system restoration is a highly complex multi-stage decision and control problem [6]. Typically, it is categorized in three stages according to different system statuses and restoration goals: a) black-start, b) system restoration, and c) load restoration [7]. In recent years, the wind power penetration has changed the power system restoration procedure needed to be undertaken after a partial or total power system blackout [8][10]. In [8], wind power units are utilized in the last restoration stage, however, the uncertain nature of wind power has been totally ignored. In order to find the optimal strategy of system restoration stage under intermittency of wind power, in [9] the application of a stochastic programming method is presented. The control system of wind turbine in black-start stage is investigated in [10]. Concentrating on the application of largescale wind farms, this paper is devoted to help the decisionmaking process in load restoration problem. Load restoration is the last restoration stage in order to recover load demand which is the final goal of power system restoration [7]. On the one hand, a relatively stable status in this stage can satisfy the voltage and frequency restriction in power system with wind farm integration. On the other hand, the objective of load restoration is to minimize the unserved load amount based on the available generation of units [7]. The proper arrangement of wind power application can lead to a full and rapid load restoration by providing extra power supply.

There are three major issues for determining load restoration strategy [11]-[14]: i) location of load to restore, i.e. where, ii) restorable size of load, i.e. how much, and iii) operation time, i.e. when. Several load serving strategies, as a part of online restoration decision support tool, are presented in [6]. In [11] and [15], fast calculation technologies of load pickup amount are proposed according to the voltage and frequency limitations. A wide-area monitoring system (WAMS) based on-line load restoration is presented in [12] and [13]. The state-of-the-art of bulk system load restoration is regarded to the on-line restoration based on measurement devices. However, a common problem of the above studies is their sensitivity to the system power uncertainty considering intermittent wind power. Thus, the deterministic load restoration strategy may provide infeasible solutions with wind power penetration. Accordingly, the determination of bulk system load restoration strategy needs uncertain management to handle the input random variables of the problem. Robust optimization, chanceconstrained programming and scenario-based stochastic programming are widely implemented in uncertain optimization area [16]. Nevertheless, the requirements of uncertain boundary and probabilistic distribution function prevent the former two methods to be applied on the bulk system load restoration. Scenario-based stochastic programming has been employed in the offline restoration [9]. However, it is not available for the on-line restoration since the computation efficiency is low when the number of scenarios is large. Thus, a more effective method is required in order to take the advantage of the availability of the on-line data and provide the optimal load restoration strategy in the uncertain environment.

In this paper, a novel utility-oriented load restoration scheme is established to deal with the above noticed issues. Firstly, in order to complete the restoration procedure, a stepby-step process is designed where the main concern is to solve the single-step 'where, how much and when' problem considering uncertain on-line data. Secondly, a utility-based decision-making method is proposed to obtain the preferable and optimal strategy under uncertain circumstance. According to value-at-risk (VaR) and conditional value-at-risk (CVaR) [17][18], the power deficiency threshold (PDT) and conditional power deficiency (CPD) are defined to describe the uncertain power deficiency. Thirdly, a utility-oriented optimization is formulated to determine the optimal strategy with security constraints. Furthermore, by applying the optimization advantage of CPD [19]-[20] and the linear programming of AC power flow (LPAC) method [21], the utility-oriented optimization converts into a scenario-based linear programming (LP).

The main contributions of this paper are as follows: 1) Taking into account the integration of large-scale wind farms, the proposed method fills the gap between the on-line data of uncertain condition and the optimal load restoration strategy. 2) By providing robust strategy with adjustable robustness level, the proposed utility-oriented optimization can offer extra flexibility to the system operator to make decisions in power system restoration with wind power integration. 3) Another contribution of this study is the proposed algorithm does not require any probability distribution function or uncertain set of the input random variables. Accordingly, the optimal restoration strategy can be obtained from the on-line data directly.

This paper is organized as follows: Section II gives an overview of the process of problem solving. Section III introduces uncertain load restoration through utility based method. The utility-oriented optimization in LP form is constructed in Section IV. Section V demonstrates the simulation results of the 
proposed method. Section VI gives a brief conclusion.

\section{OVERVIEW OF LOAD RESTORATION CONSIDERING WIND POWER PENETRATION}

This section presents the framework to achieve load restoration considering wind power penetration. The step-by-step process, the optimization model construction and the practical strategy application are introduced.

\section{A. Background and the process of strategy generation}

In this paper, wind power is integrated into the last stage of restoration when power system is sufficiently strong with relatively stable frequency and voltage. Since the network skeleton has been rebuilt before load restoration stage, a load block can be energized if it is picked up [7], [8] and [13]. In other hand, generating units have been restarted according to the models presented in [6]. Infrastructures for the on-line restoration are required as clarified in [12], [13]. Therefore, the on-line data of load amount and wind farm power output is available, and the states of frequency and voltage are monitored in the whole process. Each load block which needs to be restored in the transmission system (TS) represents a distribution system (DS) [22]. In this study, the load restoration amount is the robust total restoration corresponding to each load block.

The load restoration strategy is determined according to a step-by-step decision-making process. To find the optimal strategy of each step, the core concern of the step-by-step process is to build a recursive model [11], [13]-[15]. Fig. 1 presents a multi-step process to complete the load restoration problem. In each step, the on-line data is sent to the recursive module in order to provide the strategy for the current step. If the current strategy is successfully implemented, the current step moves forward to the next step. The parameters and variables of the optimization model are refreshed according to the current system restoration condition. The main key is to deploy the recursive module in each step to fulfill the procedure.

As it can be seen from Fig. 2, the three main parts of the recursive module are data preparation, model construction and strategy generation. On-line data should be prepared before decision-making in each step. The data includes two parts: i) the active and reactive power data of wind farm output and load amount for the calculation of the proposed method, ii) the real-time data of voltage and frequency for system monitoring. The optimal strategy is achieved using the on-line data. In this regards, a $100 \%$ confidence level means that the optimal result is achieved according to the possible worst scenario. The confidence level is set to a lower value as decision-making based on the worst scenario with a minimal probability is too conservative. The load restoration strategy contains the scheme of wind power deployment and load pickup. It shows the location and restorable size of load blocks, the deployment amount of wind farms and the load pickup time at the current step.

The logical process of constructing the utility-oriented optimization is illustrated in Fig. 3. It demonstrates how the main techniques deal with load restoration problem considering uncertainty, and how the initial problem transforms into the LP

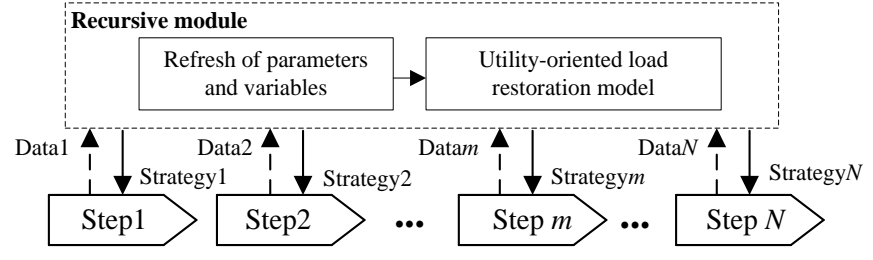

Fig. 1. The step-by-step process of utility-oriented load restoration.

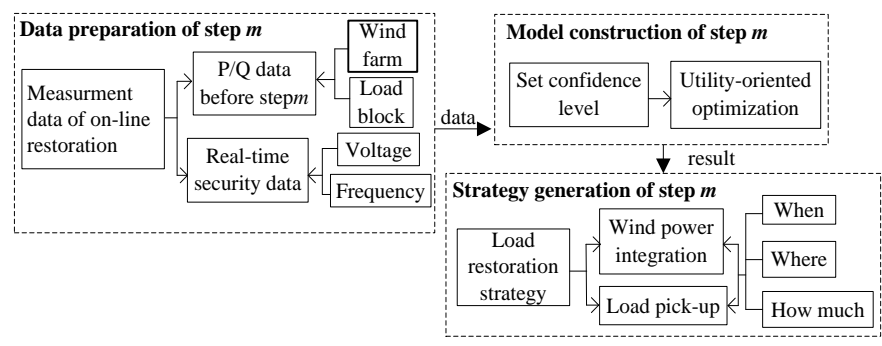

Fig. 2. Configuration of the recursive module.

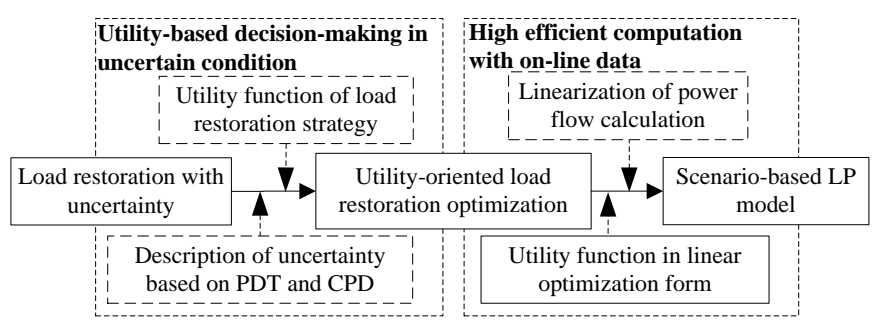

Fig. 3. The logical process of utility-oriented optimization technique

model. The 'utility-based decision-making' part and 'high efficiency computation' part are respectively detailed introduced in section IV and section V.

\section{B. Real-life load restoration strategy application}

The system operators prefer to make the decisions focusing on the worse-case conditions as the power system is generally vulnerable in restoration process [12]. Accordingly, in a conservative way, the wind farms are modeled as the fluctuating active power suppliers with the zero reactive power injection. Furthermore, in order to remain sufficient reserve in power system and to avoid some serious events such as wind power ramps under extreme weather [23], the absolute power constraint is suggested for the active power control of wind farms. Therefore, the output power of wind farm is a stochastic variable limited by the predetermined upper value.

In practice, while the wind farms are tripped off in the blackout event [2]-[3], they need some time to be started up, integrated and ramped up to the expected output powers. The process takes several minutes, thus, an operation method is proposed in Fig. 4 to apply the optimal restoration strategy.

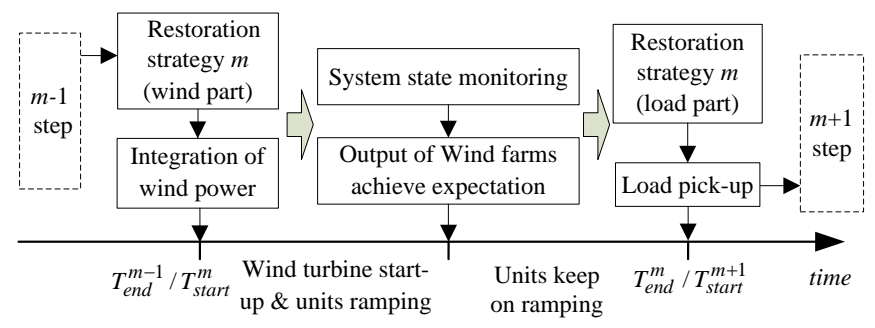

Fig. 4. Operation of the restoration strategy in each step. 
As shown in Fig. 4, in each load restoration step, wind farms should be firstly integrated, and the loads are suggested being picked up when the monitored output of wind farms reach to their expected values. Once the load pickup is accomplished, the step will be terminated. The length of each step correlated to the generation availability of the units. The on-line data not only provides input data for the optimization framework, but also helps achieve the application of strategy by checking the system frequency and voltage. In this way, the wind farms can be successfully integrated, and their power output amount can be monitored to ensure they reach to the expected values. Note that although the wind power integration and load pickup have separate operations as can be seen from Fig. 4, the strategy comes from a unified optimization illustrated in Fig. 2. The essence of the strategy application is to do all the optimization simultaneously and apply the strategies separately.

\section{LOAD RESTORATION PROBLEM BASED ON UTILITY METHOD}

This section defines several mathematical variables to formulate the utility function of load restoration strategy. The main purpose of this section is to construct the utility-based decision-making framework for the uncertain load restoration.

\section{A. Description of uncertainty and risk in load restoration procedure}

Since supply demand balance is of vital importance in the load restoration procedure, the stochastic active power is generally regarded as the core stone of the load restoration uncertainty. Thus, uncertain active power is emphasized and described by PDT and CPD which present the physical meanings of the mathematical expression of $\mathrm{VaR}$ and $\mathrm{CVaR}$, respectively.

Let us consider that $f\left(\boldsymbol{X}_{m}, \boldsymbol{Y}\right)$ is the value of system uncertain power deficiency in step $m$. It can be determined by the decision vector $\boldsymbol{X}_{m}$ and the stochastic power vector $\boldsymbol{Y}$.

$$
\begin{aligned}
f\left(\boldsymbol{X}_{m}, \boldsymbol{Y}\right) & =\boldsymbol{X}_{m} \boldsymbol{Y}^{T}=\boldsymbol{X}_{L, m} \boldsymbol{Y}_{L}^{T}-\boldsymbol{X}_{W, m} \boldsymbol{Y}_{W}^{T} \quad x_{m, i} \in(0,1) \\
& =x_{m, 1} y_{1}+\ldots+x_{m, s} y_{s}-x_{m, s+1} y_{s+1}-\ldots-x_{m, n} y_{n}
\end{aligned}
$$

$\boldsymbol{X}_{L}$ is the vector of load pickup decision variables while $\boldsymbol{X}_{W}$ presents the decision vector of wind farms. These values indicate the recovery rate of each load block and the implementation rate of the expected output power of each wind farm, respectively. $\boldsymbol{X}_{m}=\left[\boldsymbol{X}_{L, m}, \boldsymbol{X}_{W, m}\right]$ denotes the decision-making portfolio in step $m . s$ and $(n-s)$ denote the number of load block and wind farm, correspondingly. $\boldsymbol{Y}=\left[\boldsymbol{Y}_{L}, \boldsymbol{Y}_{W}\right]$ contains stochastic power vector of load blocks and wind farms.

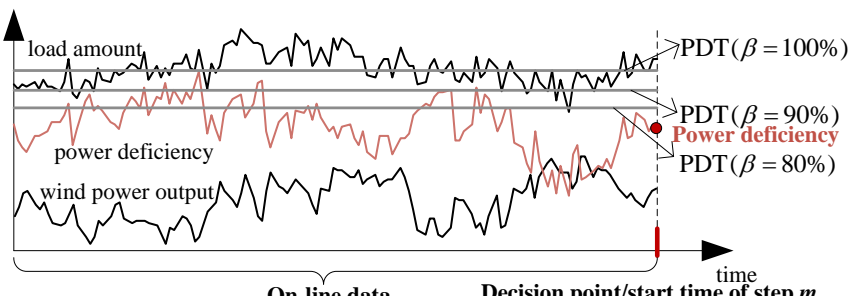

Fig. 5. The meaning of PDT with different confidence level.
For each $\boldsymbol{X}_{m}, f\left(\boldsymbol{X}_{m}, \boldsymbol{Y}\right)$ is the system random power variable with a specific probability distribution related to $Y$. The probability density function of $f\left(\boldsymbol{X}_{m}, \boldsymbol{Y}\right)$ is donated by $\rho(\boldsymbol{Y})$. Note that the proposed method doesn't need to deploy the distribution and density functions, and they are just defined here for the sake of explanation. Based on the above definitions, the probability of the system uncertain power deficiency $f\left(\boldsymbol{X}_{m}, \boldsymbol{Y}\right)$ not exceeded from the threshold $\alpha$ is provided in step $m$ as shown in (2).

$$
\Psi\left(\boldsymbol{X}_{m}, \alpha\right)=\int_{f_{m}\left(\boldsymbol{X}_{m}, \boldsymbol{Y}\right) \leq \alpha} \rho(\boldsymbol{Y}) d y
$$

$\Psi\left(\boldsymbol{X}_{m}, \alpha\right)$ is the cumulative distribution function associated with $\boldsymbol{X}_{m}$. Assuming $\Psi\left(\boldsymbol{X}_{m}, \alpha\right)$ is continuous with respect to $\alpha$, PDT and CPD values with any confidence level and load restoration strategy $\boldsymbol{X}_{m}$ are yielded as follows:

$$
\begin{gathered}
\text { PDT : } \alpha_{\beta}\left(\boldsymbol{X}_{m}\right)=\min \left\{\alpha \in R: \Psi\left(\boldsymbol{X}_{m}, \alpha\right) \geq \beta\right\} \\
\text { CPD }: \Phi_{\beta}\left(\boldsymbol{X}_{m}\right)=\frac{1}{1-\beta} \int_{f_{m}\left(\boldsymbol{X}_{m}, \boldsymbol{Y}\right) \geq \alpha_{\beta}\left(\boldsymbol{X}_{m}\right)} f\left(\boldsymbol{X}_{m}, \boldsymbol{Y}\right) \rho(\boldsymbol{Y}) d y
\end{gathered}
$$

PDT extracts the left endpoint of the corresponding nonempty interval of $\alpha$ subject to $\Psi\left(\boldsymbol{X}_{m}, \alpha\right)=\beta$, and it is the threshold of the uncertain power deficiency with confidence level $\beta$ in Fig. 5. CPD is the conditional expectation of uncertain power deficiency exceeding PDT, or the expectation of uncertain power deficiency beyond $\beta$. CPD is employed to construct a coherent risk measure $f_{r i s}$ [24]. For the same strategy, the value of (5) is influenced by the confidence level $\beta$.

$$
f_{\text {ris }}\left(\boldsymbol{X}_{m}\right)=\Phi_{\beta}\left(\boldsymbol{X}_{m}\right)-\boldsymbol{X}_{m} \boldsymbol{E}^{T}
$$

$\boldsymbol{E}=\left[\boldsymbol{E}_{L}, \boldsymbol{E}_{W}\right]$ denotes the expected load amount and wind farm output expectations. Since most security violations are due to power unbalance, the uncertain active power deviation $f_{\text {ris }}$ of a load restoration strategy can be considered as a risk index. Considering the goal of load restoration, the return $f_{\text {ret }}$ can be defined as a load recovery expectation of the same strategy as follows:

$$
f_{r e t}\left(\boldsymbol{X}_{m}\right)=\boldsymbol{w}_{L}\left(\boldsymbol{X}_{L, m}^{T} \boldsymbol{E}_{L}\right)
$$

where $\boldsymbol{w}_{L}$ is the weight set. The critical load receives the high weight factor in $\boldsymbol{w}_{L}$.

The risk and return of strategies present the quality of the load restoration strategy, while the security and efficiency refer to the effect of strategy application. The load restoration strategy with high risk value may lead to large power unbalance, and it is easy to cause security violation. The strategy with high return value associates with the larger amount of load recovery, by this, the efficiency of load restoration is improved. The restoration process can be secure and efficient if the restoration strategy has low risk value and high return value.

\section{B. Construction of utility function}

The utility function of load restoration strategy is defined in this paper to describe the preference of decision-maker [25]. A greater utility value is assigned to the more preferred strategy. In the load restoration procedure, the decision maker should be risk averse since power uncertainty will violate system security. In the other hand, the higher return is preferred for the faster 
recovery of load. Taking return and risk into account, the utility function is described as follows:

$$
\mathrm{U}\left(\boldsymbol{X}_{m}\right)=(1-c) f_{r e t}\left(\boldsymbol{X}_{m}\right)-c f_{r i s, \beta}\left(\boldsymbol{X}_{m}\right)
$$

with $c$ as the risk-averse coefficient tuned by system status (frequency, voltage deviation and load recovery state).

$$
\begin{aligned}
& c=\frac{k_{1}\left|\Delta f_{m}\right|}{\Delta f_{\max }}+\frac{k_{2} \sum\left|\Delta V_{m, i}\right|}{n \Delta V_{\max }}+\frac{k_{3}\left|\boldsymbol{X}_{L, m-1} \boldsymbol{E}_{L}^{T}-\operatorname{sum}\left(\boldsymbol{E}_{L}\right)\right|}{\operatorname{sum}\left(\boldsymbol{E}_{L}\right)} \\
& \left(k_{1}+k_{2}+k_{3}=1\right)
\end{aligned}
$$

The philosophy of utility function is to make a tradeoff between load recovery efficiency and security. The return-risk form has the same efficient frontier with the form that pursues the minimum risk with lower limit of return or maximum return with the upper limit of risk [24]. There must be an indifferent curve (IDC) set of utility with different constant $l$ according to (9).

$$
l=(1-c) f_{r e t}\left(\boldsymbol{X}_{m}\right)-c f_{r i s, \beta}\left(\boldsymbol{X}_{m}\right)
$$

The optimal load restoration strategy holds the largest value of utility within the feasible region, and it is located in the place where the indifference curve is tangent to the efficient return-risk frontier [25].

\section{Special constraint for the application of utility function}

A formula transformation of CPD is carried out since the mathematical expression (4) is intractable. According to (4), $\mathrm{CPD}$ can be represented as follows:

$$
\begin{array}{r}
F_{\beta}\left(\boldsymbol{X}_{m}, \alpha\right)=\alpha+\frac{1}{1-\beta} \int_{y \in R^{m}}\left[f\left(\boldsymbol{X}_{m}, \boldsymbol{Y}\right)-\alpha\right]^{+} \rho(y) d y \\
{[Z]^{+}=\max \{0, Z\}}
\end{array}
$$

The following equations can be yielded [19]:

$$
\begin{gathered}
\min _{\alpha \in R} F_{\beta}\left(\boldsymbol{X}_{m}, \alpha\right)=\Phi_{\beta}\left(\boldsymbol{X}_{m}\right) \\
\min _{\left.\boldsymbol{X}_{m}, \alpha\right) \in X \times R} F_{\beta}\left(\boldsymbol{X}_{m}, \alpha\right)=\min _{\boldsymbol{X}_{m} \in X} \Phi_{\beta}\left(\boldsymbol{X}_{m}\right)
\end{gathered}
$$

The extracted result of $\alpha$ in (12) is PDT. That is why PDT can be a by-product of CPD optimization computation.

To deal with the integral part and the intractable analytical expression of $\rho(\boldsymbol{Y})$, the following approximation is deployed.

$$
F_{\beta}^{*}\left(\boldsymbol{X}_{m}, \alpha\right)=\alpha+\frac{1}{M(1-\beta)} \sum_{k=1}^{M}\left[f\left(\boldsymbol{X}_{m}, \boldsymbol{Y}_{k, m}\right)-\alpha\right]^{+}
$$

where $\left[\boldsymbol{Y}_{1, m}, \ldots, \boldsymbol{Y}_{k, m}, \ldots, \boldsymbol{Y}_{M, m}\right]$ is the collection of stochastic power sampling in step $m$. Based on (4)-(7), the utility function can be written as follows:

$$
\mathrm{U}\left(\boldsymbol{X}_{m}\right)=\sum_{i=1}^{s}\left[(1-c) w_{L, i}+c\right] x_{L, m, i} E_{L, i}+c \sum_{i=1}^{n-s} x_{W, m, i} E_{W, i}-c F_{\beta}^{*}\left(\boldsymbol{X}_{m}, \alpha_{\beta}\right)
$$

Assuming the number of $\boldsymbol{Y}_{k}$ subjected to $f_{m}\left(\boldsymbol{X}_{m}, \boldsymbol{Y}\right)-\alpha>0$ is $g$ and they are indicated by $\boldsymbol{Y}^{*}$, the partial derivative of $\mathrm{U}$ with respect to $x_{L, m, i}$ can be calculated as follows:

$$
\frac{\partial \mathrm{U}}{\partial x_{L, m, i}}=\left[(1-c) w_{L, i}+c\right] E_{L, i}-\frac{c \sum_{j=1}^{g} y_{j, i}^{*}}{M(1-\beta)}-\left(1-\frac{c g}{M(1-\beta)}\right) \frac{\partial \alpha_{\beta}}{\partial x_{L, m, i}}
$$

The value of (15) depends on the sampling condition of $\boldsymbol{Y}$ and the effect of random variable $x_{i}$ on the joint uncertain power threshold PDT. It is noteworthy that the increase of $x_{i}$ does not always improve the utility value since the value of variable computed by (15) may be zero, negative or positive. In other words, the decision maker may not choose to pick up load according to utility based method. This phenomenon is unrealistic in actual load restoration procedure. Thus, a load pickup amount constraint (16) is indispensable to guarantee the proceeding of load restoration.

$$
\left\{\begin{array}{l}
\left(\boldsymbol{X}_{L, m}-\boldsymbol{X}_{L, m-1}\right) \boldsymbol{E}_{L}^{T} \geq b \sum_{i=1}^{N_{G}}\left(P_{G, i, m}-P_{G, i, m-1}\right) \\
x_{L, m, i} \geq x_{L, m-1, i}
\end{array}\right.
$$

where $P_{G, i, m}$ denotes the generation of unit $i$ in step $m, N_{G}$ is the number of unit, and $b$ is a constant in range of $(0,1)$. This formula means load pickup amount in each step should meet at least a certain percentage of generation increment.

\section{UTILITY-ORIENTED OPTIMIZATION IN LINEAR FORM}

In order to obtain the optimal strategy, a scenario-based LP model in terms of the utility-oriented optimization is introduced. After which, the application of on-line data is presented.

\section{A. Construction of the utility-oriented optimization}

The optimal load restoration strategy can be determined by optimizing utility function within load restoration constraints. Formula (17)-(30) make up the initial form of utility-oriented optimization model in step $m$.

1) Objective function: The utility-oriented load restoration approach maximizes the utility value (7). By replacing the CPD part of (5) by (13), the objective function (17) becomes:

$$
\max _{\beta,\left(\boldsymbol{X}_{m}, \alpha_{m}, t_{m}\right) \in X \times R \times R}\left\{\begin{array}{l}
\sum_{i=1}^{s}\left[(1-c) w_{L, i}+c\right] x_{L, m, i} E_{L, i}+c \sum_{i=1}^{n-s} x_{W, m, i} E_{W, i} \\
-c\left[\alpha_{m}+\frac{1}{M(1-\beta)} \sum_{k=1}^{M}\left[f\left(\boldsymbol{X}_{m}, \boldsymbol{Y}_{k}\right)-\alpha_{m}\right]^{+}\right]
\end{array}\right\}
$$

2) Power supply constraints: The single-step model of generating unit is presented in (18) as a part of the multi-step generator model proposed in [6]. It means unit $i$ ramps up with rate $r_{i}$ between the upper and lower limits. Formula (19) ensures that power supply satisfies the threshold of uncertain power deficiency (PDT). Constraint (20) makes operating time (load pickup time) $t_{m}$ within reasonable time interval. $T_{\min , m}$ is taken into account to guarantee the availability of wind farm output and the suitability of system status for load pickup. $T_{\max }$ is the upper bound of the time interval considered for the load restoration step.

$$
\begin{gathered}
P_{G, m, i}=\max \left\{P_{G, m-1, i}+r_{i}\left(t_{m}-t_{m-1}\right), P_{G, i, \min }\right\} \text { or } \\
\min \left\{P_{G, m-1, i}+r_{i}\left(t_{m}-t_{m-1}\right), P_{G, i, \max }\right\} i \in G \\
\sum_{i \in G} P_{G, m, i}=\alpha_{m} \\
t_{m-1}+T_{\min , m} \leq t_{m} \leq t_{m-1}+T_{\max }
\end{gathered}
$$

3) Power balance constraints: At each step, the active and reactive power balance constraints corresponded to the AC power flow calculation should be satisfied using (21)-(26).

$$
\begin{gathered}
p_{m, i}=-x_{L, m, i} E_{L, i}(\forall i \in L) \text { or } x_{W, m, i} E_{W, i}(\forall i \in W) \\
\quad \operatorname{orP}_{G, m, i}-x_{L, m, i} E_{L, i}(\forall i \in G)
\end{gathered}
$$




$$
\begin{aligned}
q_{m, i}= & -x_{L, m, i} q_{L, i}(\forall i \in L) \\
& o r-x_{L, m, i} q_{W, i}(\forall i \in W) \\
p_{m, i}= & \sum_{\langle i, j\rangle \in L i n e} p_{m, i j} \quad \forall i \in N \\
q_{m, i}= & \sum_{\langle i, j\rangle \in \text { Line }} q_{m, i j} \quad \forall i \in N \\
p_{m, i j}= & V_{i}^{2} g_{i j}-V_{i} V_{j}\left(g_{i j} \cos \theta_{i j}+b_{i j} \sin \theta_{i j}\right) \forall i, j \in N \\
q_{m, i j}= & -V_{i}^{2} b_{i j}-V_{i} V_{j}\left(g_{i j} \sin \theta_{i j}-b_{i j} \cos \theta_{i j}\right) \forall i, j \in N
\end{aligned}
$$

4) Security constraints: Voltage, branch flow and frequency limitations are posed in (27), (28) and (29), respectively. In practice, the reactive power compensation is usually applied in the substations to avoid transmitting reactive power through the transmission lines. Therefore, active power limit can be taken as the branch power flow limit as the reactive power is quite small [13]. If needed, a conservative constraint with the reactive power limitation $\left(q_{\max }^{2}=\boldsymbol{s}_{\max }^{2}-p_{\max }^{2}\right)$ can be added to the problem formulation to maintain the linear form. Constraint (29) is a linear frequency limit considering the total capacity of restored generators $S$ and their frequency response constant $\varepsilon$ [28]. Especially, (29) and (30) are reserve constraints required in restoration process [4]. Formula (29) is considered to provide the dynamic reserve which consists of governor response of generators, and (30) ensures spinning reserve which survives the largest loss of energy contingency [14].

$$
\begin{aligned}
& V_{i, \text { min }}<V_{i}<V_{i, \max } \quad \forall i \in N \\
& -p_{i j \max }<p_{m, i j}<p_{i j \max } \quad \forall i \in N \\
& \left\{\begin{array}{l}
\Delta f_{L, m}=\left|\left(\boldsymbol{X}_{L, m}-\boldsymbol{X}_{L, m-1}\right) \boldsymbol{E}_{L}^{T} / \sum_{j \in G, j \neq i} \frac{S_{j, m}}{\varepsilon_{j}}\right| \leq \Delta f_{\max } \\
\Delta f_{W, m}=\left|\left(\boldsymbol{X}_{W, m}-\boldsymbol{X}_{W, m-1}\right) \boldsymbol{E}_{W}^{T} / \sum_{j \in G, j \neq i} \frac{S_{j, m}}{\varepsilon_{j}}\right| \leq \Delta f_{\max } \quad \forall i \in G
\end{array}\right. \\
& P_{G, m, i} \leq \sum_{j \in G, j \neq i}\left(P_{G, m, j, \max }-P_{G, m, j, \min }\right)+\sum_{j \in G, j \neq i}\left(P_{G, m, j}\right) \\
& -\boldsymbol{X}_{L, m} \boldsymbol{E}_{L, m}^{T}+\boldsymbol{X}_{W, m} \boldsymbol{E}_{W, m}^{T} \quad \forall i \in G
\end{aligned}
$$

\section{B. Linearization of utility-oriented optimization}

There are two parts in the optimization process which make the initial model computation intractable: the objective function (17) and the power flow constraints (21)-(26). Thus, they have to be transformed into linear form. Set auxiliary variable $u_{k}(k=1, \ldots, M)$, and $u_{k}=\left[f\left(\boldsymbol{X}_{m}, \boldsymbol{Y}_{k}\right)-\alpha_{m}\right]^{+}$. Formula (17) can be transformed into the linear form provided in (31)-(33).

$$
\begin{aligned}
& \max _{\beta,\left(\boldsymbol{X}_{m}, \alpha_{m}, t_{m}\right) \in X \times R \times R}\left\{\begin{array}{l}
\sum_{i=1}^{s}\left[(1-c) w_{L, i}+c\right] x_{L, m, i} E_{L, i}+c \sum_{i=1}^{n-s} x_{W, m, i} E_{W, i} \\
-c\left[\alpha_{m}+\frac{1}{M(1-\beta)} \sum_{k=1}^{M} u_{k}\right]
\end{array}\right. \\
& \qquad \begin{array}{l}
\text { s.t. } \\
\quad u_{k} \geq 0 k=1, \ldots, M \\
\quad u_{k} \geq f\left(\boldsymbol{X}_{m}, \boldsymbol{Y}_{k}\right)-\alpha_{m} k=1, \ldots, M
\end{array}
\end{aligned}
$$

So far, the only nonlinear part is the power flow constraints. A LPAC method for load restoration is employed to achieve linear approximation of AC power flow [21]. The main aspects of LPAC are the polyhedral relaxation of cosine function and the linearization using Taylor series expansion. Besides, approximating $\sin \theta$ by $\theta$ in transmission system with small phase angle different should be conducted. Taking advantage of the above techniques, the power flow related constraints (23)-(26) can be replaced by (34)-(38).

$$
\begin{array}{r}
V_{i}=1+\delta_{i} \quad \delta_{i}(\mathrm{p} . \mathrm{u} .) \in\left(-V_{i},+\infty\right) \\
p_{i j}=g_{i j}-g_{i j} \cos ^{*} \theta_{i j}-b_{i j}\left(\theta_{i}-\theta_{j}\right) \\
q_{i j}=-b_{i j}-g_{i j}\left(\theta_{i}-\theta_{j}\right)+b_{i j} \cos ^{*} \theta_{i j}-b_{i j}\left(\delta_{i}-\delta_{j}\right) \\
\cos ^{*} \theta_{i j} \geq \cos \theta_{\Delta}^{0} \\
\cos ^{*} \theta_{i j} \leq-\sin \left(v d-\theta_{\Delta}^{0}\right)\left(\theta_{i}-\theta_{j}-v d+\theta_{\Delta}^{0}\right)+\cos \left(v d-\theta_{\Delta}^{0}\right) \\
d=2 \theta_{\Delta}^{0} /(2 h+1) \quad v=1,2, \ldots, 2 h
\end{array}
$$

\begin{tabular}{|c|c|}
\hline \multicolumn{2}{|c|}{$\begin{array}{l}\text { Desition variables : } \boldsymbol{X}_{m}, t_{m} \\
\text { objective (31) }\end{array}$} \\
\hline s.t. $(1),(8),(32)-(33)$ & Untility related constraint \\
\hline (16) & Restoration proceeding constraint \\
\hline (18)-(19) & Power supply constraint \\
\hline (20) & Operation time constraint \\
\hline \multicolumn{2}{|c|}{$(21)-(23),(34)-(38)$ Power balance constrains } \\
\hline$(27)-(30)$ & Security constrains \\
\hline
\end{tabular}

The exactness of LPAC was verified in [21] and [28], and these references demonstrate the computation error is acceptable for restoration process.

The final scenario-based LP model of the utility-oriented optimization is shown in (39). The decision variables of load restoration strategy are load pickup and wind power application scheme $\boldsymbol{X}_{m}$ and the operation time $t_{m}$. In addition to the decision variables, the result of the model proposed in (39) includes the by-product variable $\alpha_{m}$ corresponding to the value of PDT with confidence level $\beta$.

\section{Scenario generation and special model}

The utility-oriented optimization is a scenario-based LP approach. A scenario is a system restoration realization comprising recovery amount of each load block and output amount of each wind farm at a time point. The initial data can be provided using the on-line measurement devices and the final data is obtained through the application of forecasting methods or special model [15].

\section{1) Scenario generation}

Based on the on-line data in recent steps, a set of load amount and wind power output in the current step can be obtained using nonparametric or parametric forecasting methods. Final data for step $m$ consists of data matrix $\boldsymbol{P}_{m}$.

$$
\boldsymbol{P}_{m}=\left[\left(\boldsymbol{P}_{m}^{1}\right)^{\mathrm{T}}, \ldots\left(\boldsymbol{P}_{m}^{k}\right)^{\mathrm{T}}, \ldots\left(\boldsymbol{P}_{m}^{M}\right)^{\mathrm{T}}\right]=\left[\begin{array}{ccccc}
P_{m, 1}^{1} & \ldots & P_{m, 1}^{k} & \ldots & P_{m, 1}^{M} \\
P_{m, 2}^{1} & \ldots & P_{m, 2}^{k} & \ldots & P_{m, 2}^{M} \\
\ldots & \ldots & \ldots & \ldots & \ldots \\
P_{m, n}^{1} & \ldots & P_{m, n}^{k} & \ldots & P_{m, n}^{M}
\end{array}\right]
$$

where $M$ is the number of scenarios. $P_{m, i}^{k}$ is the active power of node $i$ at time $k$ in step $m .\left(\boldsymbol{P}_{m}^{k}\right)^{\mathrm{T}}=\boldsymbol{Y}_{k}$ is a scenario which presents the load recovery and wind farm output condition of the 
entire system at time $k$. Then, the auxiliary variable $u_{k}$ in the optimization model (39) can be written as follows:

$$
u_{k}=\left[\boldsymbol{X}_{m}\left(\boldsymbol{P}_{m}^{k}\right)^{\mathrm{T}}-\alpha_{m}\right]^{+} k=1, \ldots, M
$$

Define $P_{W, s i g, m}$ in (42) as the sign of wind power available since wind farms output cannot reach to their expected values immediately. The lower limit of the operation time $T_{\min }$ is (43).

$$
\begin{gathered}
P_{W, s i g, m}=\sum_{k=1: M} \sum_{i=1: n-s} P_{W, m, i}^{k} / M \\
T_{\min , m}=\frac{g T_{\text {max }, m}}{M} \min \left\{g: \sum_{k=1: ;} \sum_{i=1: n-s} P_{W, m, i}^{k} / g \geq P_{W, s i g, m}\right\}
\end{gathered}
$$

\section{2) Cold load pickup model}

Cold load pickup will lead to a significant increase of load amount. It will bring about serious power deficiency and even result in security problems if not fully prepared. Therefore, the cold load pickup phenomenon needs to be carefully dealt with in the load restoration procedure [11], [14]-[15]. Cold load pickup, which is influenced by temperature, outage duration and etc., is a complex process that is hard to be predicted. Particularly, a piecewise linear model (44) is employed to present the ramping behavior of cold load pickup [15]. The expression of reactive power is identical to the active power.

In (44), $P_{0}$ presents the normal load amount before the blackout occur, $t_{0}$ is the time point of cold load picking up, [ $t_{0}$, $\left.t_{0}+\Delta t_{1}+\Delta t_{2}\right]$ is the time interval of cold load behavior. All the parameters used in (44) can be identified from measurement data that are gathered during large-scale rotating interruptions [15]. Considering the gradual decreasing characteristic of the cold load pickup amount in (44), the largest amount at the start point of each load restoration step is applied in (39). Assuming some part of cold load block $i$ has been picked up at the first step and the third step, the current amount in step $m(m>3)$ would be formulated by (45).

$$
\begin{aligned}
& P_{C L}(t)= \begin{cases}0 & 0 \leq t \leq t_{0} \\
K P_{0} & t_{0}<t \leq t_{0}+\Delta t_{1} \\
K P_{0}-\frac{K P_{0}-P_{0}}{\Delta t_{2}}\left(t-t_{0}-\Delta t_{1}\right) & t_{0}+\Delta t_{1}<t \leq t_{0}+\Delta t_{1}+\Delta t_{2} \\
P_{0} & t>t_{0}+\Delta t_{1}+\Delta t_{2}\end{cases} \\
& P_{C L, i, m}=x_{C L, i, 1} P_{C L, i}\left(t_{2}+t_{3}+\ldots+t_{m-1}\right) \\
& +\left(x_{C L, i, 3}-x_{C L, i, 2}\right) P_{C L, i}\left(t_{4}+t_{5}+\ldots+t_{m-1}\right) \\
& +\left(x_{C L, i, m}-x_{C L, i, m-1}\right) P_{C L, i}\left(t_{0}\right)
\end{aligned}
$$

\section{CASE STUdY}

\section{A. Case Information}

The first test system is IEEE-30 bus test system. Five wind farms are installed in the nodes 15, 17, 20, 24 and 30 with the rated output power 10MW, 20MW, 20MW, 10WM and $30 \mathrm{MW}$, respectively. Wind power accounts for $31.03 \%$ of total system capacity. The second test system is based on the practical Shandong northeast power grid. It has 30 major plants, 17 large-scale wind farms, 177 nodes and 263 transmission lines with $220 \mathrm{kV}$ and $500 \mathrm{kV}$ voltage levels. Wind power penetration level is $26.82 \%$. Load weights in $\boldsymbol{w}_{L}$ are all set as one, and the operation time limit $T_{\max }$ is $10 \mathrm{~min}$. The limits of voltage and frequency deviation are 0.1 p.u. and $0.5 \mathrm{~Hz}$, respectively. Parameters $k_{1}, k_{2}$ and $k_{3}$ are set to $0.4,0.4$ and 0.2 , respectively. To validate the proposed method, two types of data are provided: pseudorandom data and on-line data. Pseudorandom data is extracted from the pseudorandom variables which are the Weibull PDF with shape parameter 2 and Gaussian distribution for wind speed and load amount, respectively. The on-line data used in the real world system is provided by the operation center of Shandong province and measured in every second in 2013. The on-line data has more accurate description of the system condition than pseudorandom one. In 10,000 set of pseudorandom data, the average standard deviations from expected values of wind farm output, load amount and system power deficiency are $14.87 \%, 5.32 \%$ and $12.56 \%$, respectively. The corresponding standard deviations of on-line data are $7.79 \%, 2.13 \%$ and $7.12 \%$, respectively.

\section{B. Computation efficiency of the utility-oriented model}

The derived model is solved by CPLEX V12.5.0.0 using an Intel(R) Core(TM) i5-2400 CPU and 4 GB RAM. For different scenario size, the corresponding calculation time and standard deviation of PDT and CPD are listed in Table I. They are the average results of 100 times simulation runs with pseudorandom variables. The results deviation obtains from the production of pseudorandom data and linearization of the optimization model. As can be observed, with computation time in seconds and standard deviation $\sigma$ within $1 \%$, the efficiency and exactness of the utility-oriented optimization (UO) is quite acceptable and superior for the on-line load restoration.

For the sake of comparison, the scenario-based stochastic programming (SP) technique [9] is deployed with the sample size of 500, 1000 and 10,000. The computation time and corresponded standard deviation $\sigma$ of the SP method are presented in Table I. Despite a reasonable standard deviation $\sigma \%$ (less than $1 \%$ ) of the power deficiency (PD), the SP method results in heavier computational burden with respect to the UO ones under the identical scenario size. It is due to the fact that the UO method traces the threshold condition of scenarios to make decisions accordingly. In consequence, the number of constraints is increased with scenario number growth. Thus, no matter how many scenarios are deployed, the proposed method performs the LP calculation one time, whereas the number of mathematical programming of the SP method depends on the scenario size.

\section{Load restoration strategy and the influence of wind power application}

To demonstrate the influence of wind power application, Con1 with wind power and Con 2 without wind power are set up with $\beta=0.85$.

Table II presents the multi-step load strategies with or without wind power application. The first column shows the step number, while the number outside and inside of the bracket in the second and forth columns are the node number and recovery amount, respectively. Here '*' denotes node with wind farm. The time in the third and fifth columns are the operation time of the load pickup in each step. The load restoration strategy comprises the number illustrated in the outside and inside of the bracket and the operation time in each step. With the 
proposed method, different strategies can be determined according to the system condition and restoration proceeding. Even though the total steps of different conditions are identical, Con1 takes shorter execution time in the whole restoration procedure with respect to Con2. Besides, the condition with wind power application allows system operator for more load recovery in the early steps. It means more loads can be restored earlier. In summary, the proposed method takes advantage of the active power support from wind power through the proper arrangement of wind power application.

TABLE I

COMPUTATION EFFICIENCY OF THE UTILITY-ORIENTED MODEL

\begin{tabular}{c|cc|ccc|c|cc}
\hline & \multicolumn{3}{c|}{ IEEE-30 bus test system } & \multicolumn{3}{c}{ Real world system } \\
\hline Method & \multicolumn{2}{|c|}{ SP } & \multicolumn{3}{c}{ UO } & SP & \multicolumn{2}{c}{ UO } \\
\hline Scenario size & 500 & $10^{\wedge^{3}}$ & 500 & $10^{\wedge^{3}}$ & $10^{\wedge^{4}}$ & $10^{\wedge^{3}}$ & $10^{\wedge^{3}}$ & $10^{\wedge^{4}}$ \\
\hline $\begin{array}{c}\text { Computation } \\
\text { time(s) }\end{array}$ & 8.01 & 19.59 & 0.07 & 0.17 & 8.72 & 77.63 & 5.67 & 12.51 \\
PDT $(\sigma \%)$ & NA & NA & 0.53 & 0.44 & 0.11 & NA & 0.72 & 0.19 \\
CPD $(\sigma \%)$ & NA & NA & 0.59 & 0.36 & 0.10 & NA & 0.63 & 0.16 \\
$\operatorname{PD}(\sigma \%)$ & 0.47 & 0.28 & NA & NA & NA & 0.69 & NA & NA \\
\hline
\end{tabular}

TABLE II

MULTI-STEP LOAD RESTORATION STRATEGIES OF DIFFERENT CONDITIONS

\begin{tabular}{|c|c|c|c|c|}
\hline Step & $\begin{array}{c}\text { Con1 } \\
\text { node(MW) }\end{array}$ & $\begin{array}{c}\text { Con1 } \\
\text { time(min) }\end{array}$ & $\begin{array}{c}\text { Con2 } \\
\text { Node(MW) }\end{array}$ & $\begin{array}{c}\text { Con2 } \\
\text { time }(\min )\end{array}$ \\
\hline 1 & $\begin{array}{c}14(1.66) 16(3.50) 19(4.37) \\
20(4.4) 23(3.20) 24(0.16) \\
26(3.50) 29(2.4) 30(6.60) \\
15 *(5.40) 17 *(4.36) \\
20 *(3.00) 24 *(7.18) \\
30 *(6.36)\end{array}$ & 5 & $\begin{array}{c}14(0.87) 15(0.93) 18(0.91) \\
19(9.50) 23(1.81) 24(1.62) \\
30(9.34)\end{array}$ & 8.15 \\
\hline 2 & $\begin{array}{c}10(5.80) 14(4.54) 15(4.88) \\
17(0.83) 18(3.00) 21(5.78) \\
24(6.02) 30(2.12) \\
20 *(3.08) 17 *(1.33) \\
24 *(1.48) 30 *(2.42)\end{array}$ & 9.12 & $\begin{array}{c}10(5.8) 14(5.33) 16(2.79) \\
17(1.81) 18(2.29) 20(2.20) \\
21(0.78) 29(2.03)\end{array}$ & 10 \\
\hline
\end{tabular}

\begin{tabular}{|c|c|c|c|c|}
\hline & $(2.99) 17(1.47) 18(3.40)$ & & & \\
\hline & $21(9.10)$ & & $15(3.18) 16(0.71) 17(2.81)$ & \\
\hline 3 & $24(2.47) 30(2.12)$ & 10 & $21(16.72) 23(1.39) 24(1.7)$ & 10 \\
\hline & $15 *(2.23) 17 *(2.90)$ & & $26(1.25)$ & \\
\hline & $24 *(2.58) 30 *(2.81)$ & & & \\
\hline & $15(8.53) 17(6.70) 24(0.52)$ & & $15(4.09) 17(4.38) 24(2.00)$ & \\
\hline 4 & $17 *(0.30) 20 *(1.32)$ & 5.41 & $26(1.54) 29(0.37) 30(0.80)$ & 6.44 \\
\hline & $24 *(1.58) 30 *(1.93)$ & & & \\
\hline 5 & $24(2.09)$ & 5 & $24(2.01) 26(0.13) 30(0.46)$ & 5 \\
\hline 5 & $24 *(1.14) 30 *(3.10)$ & 5 & 2.6 & 5 \\
\hline & $24(1.57)$ & & & \\
\hline 6 & $15 *(0.65) 20 *(1.74)$ & 5 & $24(1.35) 26(0.58)$ & 5 \\
\hline & $24 *(2.13)$ & & & \\
\hline
\end{tabular}

TABLE III

LOAD RECOVERY IN EACH STEP WITH DIFFERENT WIND POWER PENETRATION

\begin{tabular}{cccc}
\hline \hline Step & $\begin{array}{c}\text { Con3 } \\
\text { Restore /time }\end{array}$ & $\begin{array}{c}\text { Con4 } \\
\text { Restore /time }\end{array}$ & $\begin{array}{c}\text { Con5 } \\
\text { Restore/time }\end{array}$ \\
\hline 1 & $24.55 \mathrm{MW} / 5 \mathrm{~min}$ & $26.97 \mathrm{MW} / 5 \mathrm{~min}$ & $23.75 \mathrm{MW} / 5 \mathrm{~min}$ \\
\hline 2 & $19.45 \mathrm{MW} / 9.24 \mathrm{~min}$ & $21.72 \mathrm{MW} / 9.98 \mathrm{~min}$ & $28.30 \mathrm{MW} / 10 \mathrm{~min}$ \\
\hline 3 & $21.72 \mathrm{MW} / 10 \mathrm{~min}$ & $27.12 \mathrm{MW} / 10 \mathrm{~min}$ & $23.24 \mathrm{MW} / 10 \mathrm{~min}$ \\
\hline 4 & $12.17 \mathrm{MW} / 5.66 \mathrm{~min}$ & $11.56 \mathrm{MW} / 6.43 \mathrm{~min}$ & $13.55 \mathrm{MW} / 6.42 \mathrm{~min}$ \\
\hline 5 & $11.19 \mathrm{MW} / 5 \mathrm{~min}$ & $3.05 \mathrm{MW} / 5 \mathrm{~min}$ & $1.58 \mathrm{MW} / 5 \mathrm{~min}$ \\
\hline 6 & $1.34 \mathrm{MW} / 5 \mathrm{~min}$ & $\mathrm{NA}$ & $\mathrm{NA}$ \\
\hline \hline
\end{tabular}

The load restoration conditions with different levels of wind power penetration are shown in Table III. Excepting the initial $31.03 \%$ wind power penetration in Con3, the available output power of wind farms are further increased to $48.28 \%$ and $72.41 \%$ in Con 4 and Con5, respectively. The six restoration steps of Con3 are reduced to five steps of Con4 and Con5 through improving the wind power participation level. The total load restoration process provided in Con3 is faster than Con4, which is not the case for Con 4 and Con5. Even though the larger load demand of Con4 is recovered earlier than that of Con5, the restoration time is not improved. That is because the utility-based method pursues the best compromised solution between the risk and return under uncertain status, and the power system reserves constraints (29)-(30) also provide additional limitations in wind power application. Accordingly, the available wind power growth can benefit the restoration process, however, the improvement may not be obvious since the corresponding load restoration strategy may not employ total available wind power.

\section{Influence of the security requirement}

The utility-oriented optimization guarantees the robustness of the strategy according to a certain confidence level $\beta$ which indicates the security requirement. To compare the return-risk spectrums and the effects of the optimal strategies with different security requirement, different conditions are designed as $\beta=0.75$ (Con 1 ), $\beta=0.85$ (Con 2 ) and $\beta=0.95$ (Con3). In order to avoid the influence of the risk avoiding index $c$, it is set to 0.5 .

Fig. 6 portrays the relationship between IDC with the largest utility value and the efficient frontier of the utility function in different conditions. The subfigures (a), (b), (c) and (d) are corresponded to the four load restoration steps. Different step means discrepant restoration state before decision-making. IDCs with the largest utility value are highlighted with yellow color in Fig. 6. Since these IDCs are corresponded to the utility values of the optimal strategies, they should theoretically tangent to the efficient frontiers. This phenomenon is presented with different confidence level in each subfigure of Fig. 6. Therefore, despite the discrepancy in security requirement and system state, the proposed method can achieve the best riskreturn balanced strategy. Note that it is not strictly meet the mathematical definition of tangent due to the error of pseudorandom data.

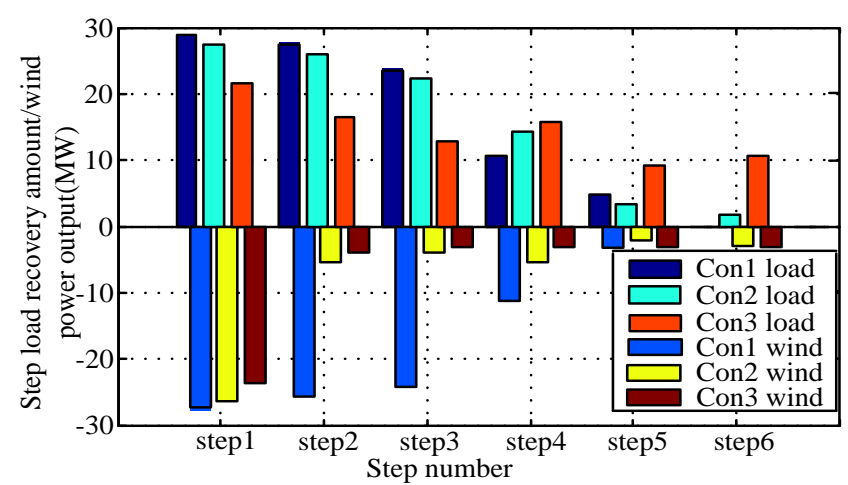

Fig. 7. Load and wind power recovery of each step with different $\beta$ 


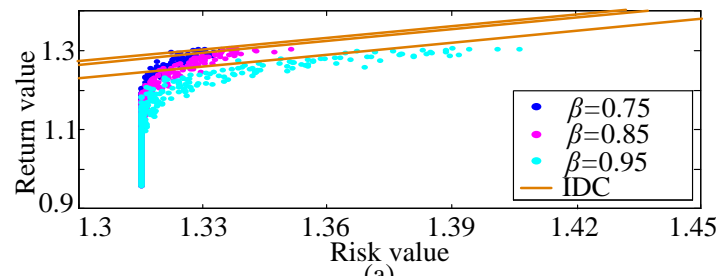

(a)

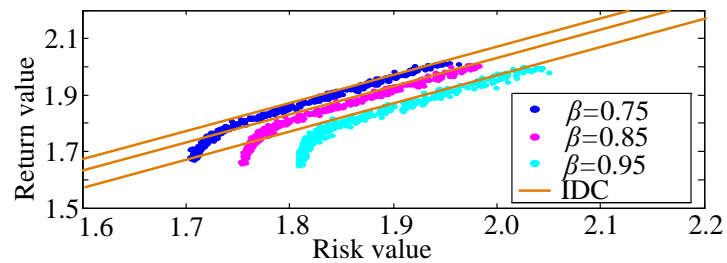

(c)

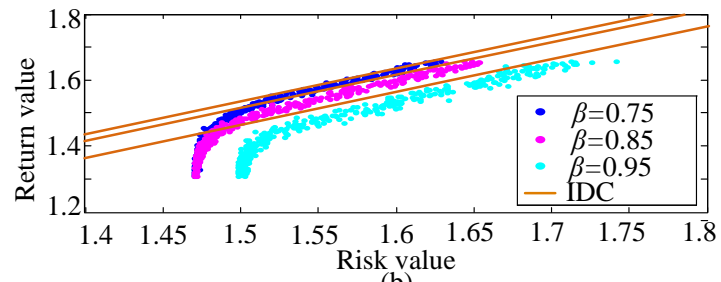

(b)

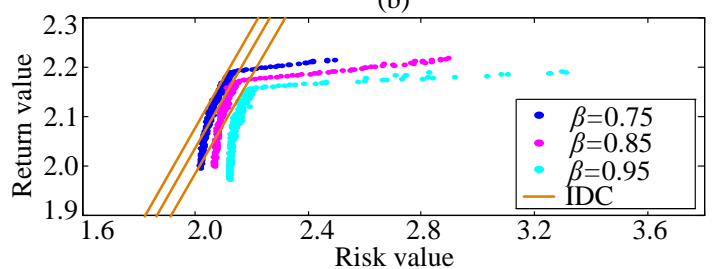

(d)

Fig. 6. Return-risk spectrums of different $\beta$ in four restoration steps

As shown in Fig. 6, all points with different colors form the efficient frontiers are associated with different $\beta$. They demonstrate the return-risk spectrums with different security requirement. For each step, the efficient frontier with higher $\beta$ is in lower right. It means the higher security requirement is with the higher risk (the risk is emphasized) while the return value is the same. This phenomenon can be explained by the definitions of PDT and CPD. For the same uncertain condition, PDT becomes larger while $\beta$ is higher in (3). Since CPD is the conditional expectation exceeding PDT, the value of CPD as well as the risk in (5) becomes larger. Thus, for identical return, the higher risk appears along with higher value of $\beta$.

A higher $\beta$ along with a higher PDT mean that the strategies should satisfy a wider range of uncertainty level, so that the strategy with stronger robustness will be selected. 'Stronger robustness' describes a more conservative strategy with less load pickup and wind power application. Fig. 7 shows the load restoration process with different value of $\beta$. As can be seen, Con1 with the lowest $\beta$ restores the highest amount of load 'Con1 load' and deploys the largest wind power value 'Con1 wind' in the early stages. On the contrary, the load recovery is more decentralized in Con3 'Con3 load' compare to others. Furthermore, the restoration time also shows the difference in conservatism. The higher $\beta$ leads to the restoration time increment, thus, the completion time of Con1, Con 2 and Con 3 are $38.38 \mathrm{~min}, 40.15 \mathrm{~min}$ and $41.27 \mathrm{~min}$, respectively. Generally, the larger $\beta$ brings about more conservative decision. That is why the proposed method can flexibly adjust the robustness according to the security requirement.

\section{E. Application of the utility-oriented model in real world grid}

Two groups of data are applied with $\beta=0.85$. The pseudorandom data with standard deviation of system power deficiency $12.56 \%$ is less exact than the on-line one with standard deficiency $7.12 \%$.

Fig. 8 demonstrates power supply and restoration conditions in four load restoration steps (a), (b), (c) and (d). The uncertain on-line data of load amount, wind power output and power deficiency are respectively presented by 'L-data', 'W- data' and 'P-data'. To deal with the uncertainty, the power supply decision according to the on-line data 'real-P-sup' and pseudorandom data 'pesu-P-sup' are both beyond the expected power deficiency 'exp-P-def'. Moreover, 'pesu-P-sup' provides greater power than 'real-P-sup' under identical security requirement. That is because less exactness of data leads to the wider range of uncertainty, consequently, PDT value is higher as it is corresponded to the uncertain power deficiency threshold. In other words, the power supply requirement based on less accuracy data tends to be more robust. Additionally, since the increase of risk index CPD follows PDT, decision-making with pseudorandom data pays more attention to the risk in restoration procedure. Therefore, as shown by the histograms in Fig. 8, with the same or larger wind power application amount, the load pickup amount 'pesu-L' with pseudorandom data tends to be lower than the one 'real-L' with on-line data.

Fig. 9 demonstrates the process of strategy application through the system frequency and voltage condition. Obviously, if the load restoration strategy is made according to the expected values of load pickup amount and wind power output, a second outage is easy to occur because of frequency violation 'exp-fre'. Robustness adjustment of two different data could be presented by the whole process. Strategies under pseudorandom data sacrifice restoration time to obtain a better performance of frequency and voltage. In the other hand, through earlier load pickup ' $*$ ' and timely application of wind power ' $\Delta$ ', the on-line data improves the efficiency of load restoration and reduces the conservation of decision-making. Since the load amount is uncertain, voltage violation exists in Fig. 9. This condition can be monitored by on-line data and remind dispatchers to apply greater reactive power compensation. Note that confidence level $85 \%$ does not mean the security constraints are satisfied with $85 \%$ probability. It refers to the decision-making process considering the worst power deficiency condition under $85 \%$ confidence level.

\section{CONCLUSION}

In this paper, a novel method for load restoration 


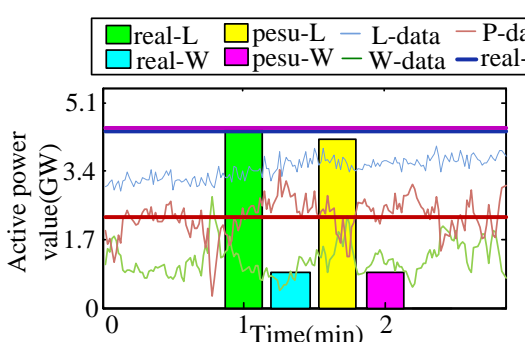

(a)

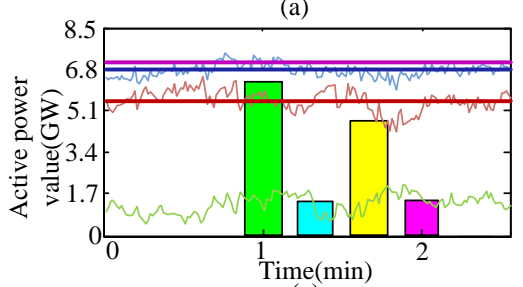

(c)

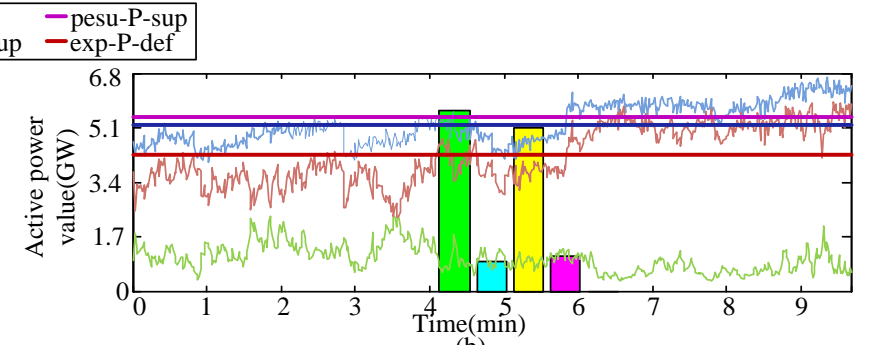

(b)

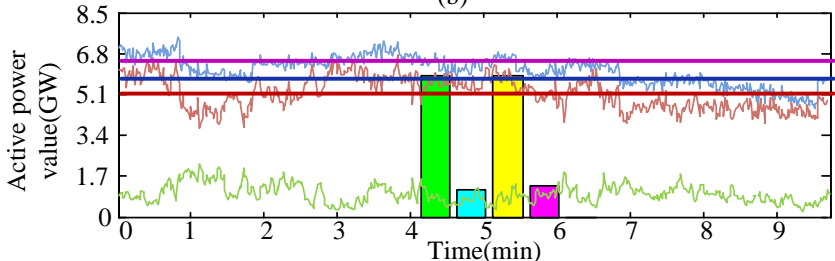

(d)

Fig. 8. Power supply and restoration conditions of each step with on-line and pseudorandom data
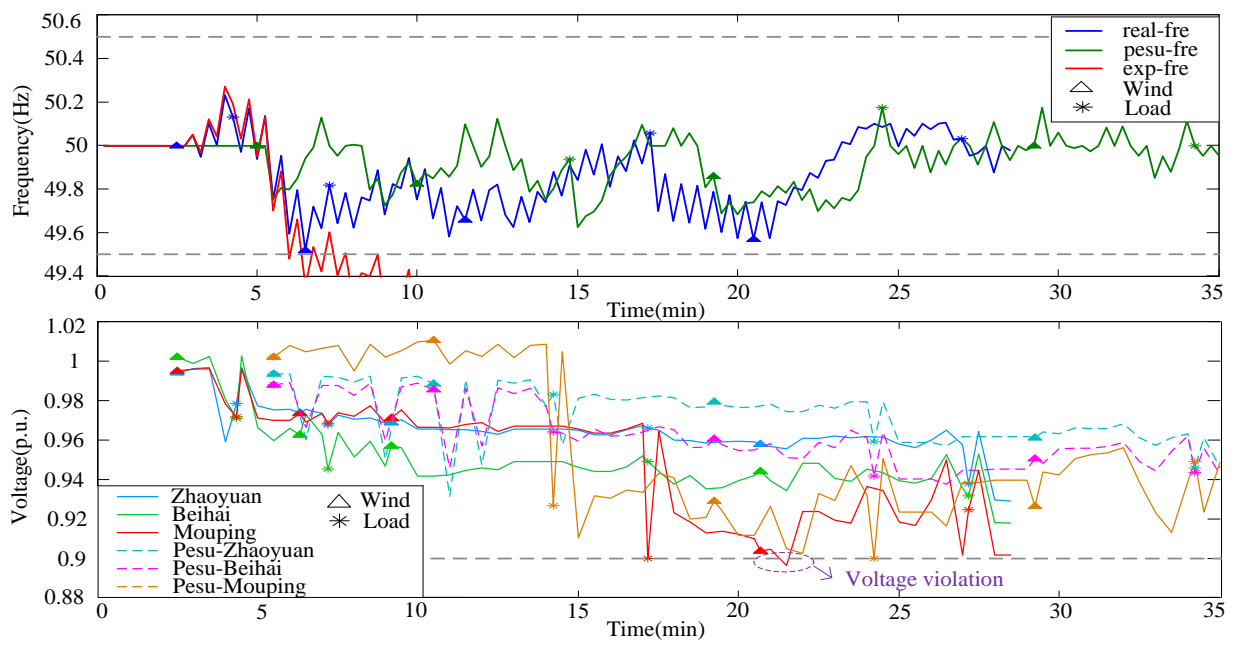

Fig. 9. Conditions of frequency and voltage in restoration procedure

considering wind power penetration is presented. A utilitybased decision-making technique is introduced to support the selection of load restoration strategy in an uncertain environment. In order to obtain the optimal strategy, a utility-oriented optimization with tractable computation is formulated. By applying the proposed model, the optimal strategy is obtained directly from the on-line measured data. Besides, the utilityoriented method performs the best tradeoff between the load restoration security and efficiency and ensures sufficient power supply with a certain confidence level. The case studies demonstrate the superior computation efficiency of the utilityoriented optimization. The optimal strategy provides properly arranged wind power application and load pickup, thereby, to speed up load restoration. In addition, the flexible adjustment of robustness is achieved by changing the confidence level or the exactness of the measured data.

This paper deals with Wind-for-Restoration problem in the last stage of restoration in the scope of uncertain optimization. The proposed framework can be extended in the future work by addressing the early stages of restoration, detailed control characteristic of renewable energies and the coordination of TS-DS restoration with binary variables.

\section{REFERENCES}

[1] R. Piwko, P. Meibom, H. Holttinen, S. Baozhuang, N. Miller, Y. Chi, and W. Wang, "Penetrating insights: Lessons learned from large-scale wind power integration," IEEE Power Energy Mag., pp. 44-52, Mar./ Apr. 2012.

[2] (Jan. 30, 2007) Final report: System Disturbance on 4 November 2006. [online]. Available: https://www.entsoe.eu/fileadmin/user_upload/_libra ry/publications/ce/otherreports/Final-Report-20070130.pdf.

[3] Black system south Australia, Mar. 2017, [Online]. Available: http://www.aemo.com.au/-/media/Files/Electricity/NEM/Market Notices and Events/Power System Incident Reports/2017/Integrated-FinalReport-SA-Black-System-28-September-2016.pdf.

[4] (Jun. 20, 2015) PJM Manual 36: System Restoration. [online]. Available: http://www.pjm.com/ /media/documents/manuals/m36.ashx

[5] (Dec. 15, 2016) IESO, Ontario power system restoration plan. [online]. Available: http://www.ieso.ca/-/media/files/ieso/documentlibrary/marke t-rules-and-manuals-library/market-manuals/systemoperations/soontpowersysrestoreplan.pdf

[6] Y. Hou, C. C. Liu, K. Sun, et al. "Computation of Milestones for Decision Support During System Restoration," IEEE Trans. Power Syst., vol. 26, no. 3, pp. 1399-1409, Aug. 2011.

[7] L.H. Fink, K. L. Liou and C. C. Liu "From Generic Restoration Actions to Specific Restoration Strategies Power system restoration dynamics issues," IEEE Trans. Power Syst., vol. 10, no. 2, pp. 745-752, May, 1995.

[8] A. M. El-Zonkoly, "Renewable energy sources for complete optimal power system black-start restoration," IET Gener. Transm. Distrib., vol. 9, no. 6, pp. 531-539, Apr. 2015. 
[9] A. Golshani, W. Sun, Q. Zhou, et al. "Incorporating Wind Energy in Power System Restoration Planning," IEEE Trans. Smart Grid, to be pulished.

[10] L. Sun, C. Peng, J. Hu, et al. "Application of Type 3 Wind Turbines for System Restoration," IEEE Trans. Power Syst., to be pulished.

[11] H. Qu, Y. Liu, "Maximizing restorable load amount for specific substation during system restoration," Int. J. Elect. Power Energy Syst., vol. 43, no. 1, pp. 1213-1220, Dec. 2012.

[12] W. Liu, Z, Lin, F. Wen, et al. "A wide area monitoring system based load restoration method." IEEE Trans. Power Syst, vol. 28, no.2, pp. 2025-2034, May 2013.

[13] A. Gholami, F. Aminifar, "A Hierarchical Response-Based Approach to the Load Restoration Problem," IEEE Trans. Smart Grid, no. 99, pp. 110, Dec. 2015.

[14] Z. Qin, Y. Hou, C. C. Liu, et al. "Coordinating generation and load pickup during load restoration with discrete load increments and reserve constraints," IET Gener. Transm. Distrib., vol. 9, no. 15, pp. 2437-2446, Nov. 2015.

[15] D. R. Medina, E. Rappold, O. Sanchez, et al. "Fast Assessment of Frequency Response of Cold Load Pickup in Power System Restoration," IEEE Trans. Power Syst, vol. 31, no. 4, pp. 3249-3256, Jul. 2016.

[16] N. Zhang, C. Kang, Q. Xia, et al. "A Convex Model of Risk-Based Unit Commitment for Day-Ahead Market Clearing Considering Wind Power Uncertainty," IEEE Trans. Power Syst, vol. 30, no. 3, pp. 1582-1592, Oct. 2014.

[17] R. T. Rockafellar, S. Uryasev, "Optimization of conditional value-atrisk," J. risk, vol. 2, pp. 21-42, Sep. 1999.

[18] R. T. Rockafellar, S. Uryasev, "Conditional value-at-risk for general loss distributions," J. banking finance, vol. 26, no. 7, pp. 1443-1471, Jul. 2002.

[19] A. Rabiee, A. Soroudi, A. Keane, "Risk-Averse Preventive Voltage Control of AC/DC Power Systems Including Wind Power Generation," IEEE Trans. Sustain. Energy, vol. 6, no. 4, pp. 1494-1505, Oct. 2015.

[20] R. Khodabakhsh, S. Sirouspour, "Optimal Control of Energy Storage in a Microgrid by Minimizing Conditional Value-at-Risk," IEEE Trans. Sustain. Energy, vol. 7, no. 3, pp. 1264-1273, Jul. 2016.

[21] C. Coffrin, P. Van Hentenryck, "A linear-programming approximation of AC power flows," INFORMS J. Comput., vol. 26, no. 4, pp. 718-734, Nov. 2014.

[22] A. Kargarian and Y. Fu, "System of Systems Based SecurityConstrained Unit Commitment Incorporating Active Distribution Grids," IEEE Trans. Power Syst, vol. 29, No.5, Mar. 2014.

[23] M. Cui, C. Feng, Z. Wang and J. Zhang, "Statistical Representation of Wind Power Ramps Using a Generalized Gaussian Mixture Model," IEEE Trans. Sustain. Energy, vol. 9, no. 1, pp. 261-272, Jan. 2017.

[24] G. C. Pflug, "Some remarks on the value-at-risk and the conditional value-at-risk," in Probabilistic Constrained Optimization, 1th ed. Dordrecht, Netherlands: Kluwer Academic Press 2000, pp. 272-281.

[25] H. R. Varian, Intermediate microeconomics: a modern approach, 8th ed. the U.S.: W.W. Norton, 2010, pp.55.

[26] P. Krokhmal, J. Palmquist, S. Uryasev, "Portfolio optimization with conditional value-at-risk objective and constraints," J. risk, vol. 4, pp. 43-68, 2002.

[27] M. M. Adibi, J. N. Borkoski, R. J. Kafka, et al., "Frequency response of prime movers during restoration," IEEE Trans. Power Syst, vol.14, no.2, pp. 751-756, May. 1999.

[28] C. Coffrin, P. Van Hentenryck, "Transmission system restoration with co-optimization of repairs, load pickups, and generation dispatch," Int. J. Elect. Power Energy Syst., vol. 72, pp. 144-154, Nov. 2015. 